\title{
LA ESCUELA: ¿DE LA SUBSIDIARIEDAD A LA SUSTITUCIÓN DE LA FAMILIA?
}

Ana Teresa López de Llergo $V$.

\section{RESUMEN}

El tema que nos ocupa se refiere a la educación en la escuela, diferenciada según el sexo. Discernir la conveniencia de las instituciones mixtas, o de las exclusivamente femeninas o masculinas. Aunque hay opiniones en pro y en contra, el estudio actual ha de considerar las características sociales que obviamente varían los esquemas tradicionales. La familia sufre las modificaciones del trabajo extradoméstico de la mujer que cambia los roles que tradicionalmente se le asignaban a ella y al varón. Otras veces, influyen políticas demográficas; esto ha reducido la natalidad y la experiencia de convivir con mayor número de personas. Hay deterioro en la fidelidad conyugal, entonces la relación con los progenitores es incompleta. La cultura contemporánea está sustituyendo el tradicional sexo por el género. Se fomenta la permisividad en las relaciones sexuales.

El panorama ofrece más sombras que luces. Sin embargo, aunque es arduo el trabajo, la familia ha de recuperar su papel de primera educadora y la escuela tomar su postura de subsidiariedad. La preparación de los maestros ha de responder a las demandas de los hijos que no cuentan con el apoyo de un buen ambiente familiar.

Es necesario ofrecer diversos tipos de escuelas: femeninas, masculinas y mixtas, para poder elegir la que más convenga a los educandos, previo estudio muy concienzudo de la personalidad y el ambiente en donde se desenvuelven. 


\section{INTRODUCCIÓN}

La familia es el ámbito natural que arropa a todo ser humano para su desenvolvimiento. Allí se convive íntimamente y, por eso, resulta más fácil el desarrollo de cada miembro, pues se cuenta con la ayuda de los seres que más nos conocen y de los que también se espera cariño.

Durante siglos lo más común, en las diversas culturas de la tierra, eran las familias numerosas, tanto por la prole que engendraban como por la cercanía e inclusión de los integrantes de la familia extensa. Sin embargo, existen muchos factores - psicológicos y sociales- que han variado la estructura familiar y, sobre todo, los roles de los progenitores. Todo ello afecta las responsabilidades de la escuela.

La institución educativa está concebida para ofrecer a la familia un servicio de subsidiariedad en la educación de los hijos, su ayuda se hace indispensable cuando los contenidos de las ciencias se enriquecen y la complejidad social exige una preparación que los padres ya no pueden proporcionar por falta de tiempo o de conocimientos especializados.

El tema que nos ocupa se refiere a la educación diferenciada, según el sexo, que puede darse en la escuela. En concreto, se trata de discernir la conveniencia de las instituciones mixtas, o de las exclusivamente femeninas o masculinas. Todo ello inmerso en las características sociales actuales que varían los esquemas tradicionales.

\section{COMPLEJIDAD DE LA FAMILIA, HOY}

Desde la segunda mitad del siglo pasado es notoria la promoción de la familia pequeña, debido a las modificaciones económicas que han exigido de la mujer un trabajo extradoméstico. No siempre el motivo es la falta de recursos, a veces es el deseo de ejercer la profesión que la capacita para desempeñar cargos fuera del hogar. Esta circunstancia varía los roles que tradicionalmente se daban a la mujer y al varón: ella en el hogar, él en el ámbito civil. Antes, había una 
clara diferencia que comprendía también el modo de sentir, planear y razonar; actualmente, como el trabajo es el mismo, hay poca diferenciación.

En muchas ocasiones los espacios habitacionales se han reducido y se dificulta acoger a una prole numerosa; otras veces influyen políticas demográficas de muy diversa ideología, que no es el caso analizar aquí. A su vez, la ciencia ofrece medios para controlar la natalidad que, aunque siempre han existido, ahora se promueven y se comercializan de tal manera que todos tienen acceso a ellos. Es tal la difusión que, desde la infancia, se asumen esos recursos como algo natural y esto ha cambiado la mentalidad de los futuros adultos.

Así las cosas, la familia está prácticamente compuesta de los padres y dos hijos, por eso no hay mucha oportunidad de convivir con diversas personalidades e incluso, cuando los hijos son del mismo sexo, tampoco aprenden a tratar a los del otro. El asunto se complica si sólo hay un hijo.

El deterioro de la fidelidad conyugal impide a los esposos cuidar el ambiente familiar y, por tanto, no se ponen los medios para evitar riñas, malos tratos, faltas de respeto y violencia. Muchas veces el desenlace es el divorcio y, más tarde, vienen las situaciones irregulares al acceder a nuevas uniones.

Cada vez son más frecuentes las familias monoparentales en donde el modelo del adulto o es femenino o masculino, con el consiguiente empobrecimiento, a no ser que vivan con tíos y abuelos. Sin embargo, la relación con los progenitores es incompleta.

La cultura contemporánea está sustituyendo el tradicional sexo por el género, de manera que la personalidad ya no se apoya en la dotación biológica sino en la influencia cultural. Los criterios que se manejan, fuertemente promovidos por la publicidad, son los de la opción de elegir el rol femenino o masculino. En muchos ambientes se va adoptando este enfoque. 
Mientras que el término sexo hace referencia a la naturaleza e implica dos posibilidades (varón y mujer), el término género proviene del campo de la lingüística donde se aprecian tres variaciones: masculino, femenino y neutro. Las diferencias entre el varón y la mujer no corresponderían, pues — fuera de las obvias diferencias morfológicas-, a una naturaleza "dada", sino que serían meras construcciones culturales "hechas" según los roles y estereotipos que en cada sociedad se asignan a los sexos («roles socialmente construidos") $)^{1}$.

Este criterio propicia el planteamiento de la conveniencia, según algunas circunstancias, de fundar una familia con dos personas del mismo sexo; y, ante la objeción de que en una unión así no habría procreación, se cuenta ahora con que pueden apoyarse en el recurso de la fecundación artificial, e incluso, en que ya algunos países conceden la adopción a parejas de este tipo. Los infantes con ese entorno tienen desdibujada la maternidad y la paternidad; la carencia de relación de alteridad procreativa fomenta la autosuficiencia en los adultos y en los jóvenes aislamiento que cuando sean adultos les dificultará la auténtica donación.

Finalmente, los medios de difusión promueven la permisividad en las relaciones sexuales, se asumen como naturales las faltas de respeto y se fomentan dependencias que impiden la madurez en las personas, y precisamente por ello, no están en condiciones de salir adelante y quedan atrapadas en este juego.

En un estudio ${ }^{2}$ para investigar la influencia de la erotización y la agresividad en el reconocimiento de la propia identidad, se tomó una muestra al azar en forma no probabilística de 138 niñas y 102 varones, la mayoría de 8 años, casi todos con un solo hermano. $48 \%$ vive con la familia nuclear; $42 \%$,

${ }^{1}$ BURGGRAF, Jutta. ¿Qué quiere decir género?, pp.9-10.

${ }^{2}$ Cfr. OÑATE GALVÁN, Rebeca. La erotización infantil en un mundo agresivizado: sus riesgos, en Aletheia, Revista anual, Número 20, pp.29-42. 
con la familia extensa, y $17 \%$ no vive con el padre. Los resultados obtenidos señalan que, en el proceso de identidad, la mayoría de las niñas se identifica con el sexo opuesto, lo que indica que la erotización provoca una tergiversación. La niña desea parecerse al padre por la relación que le liga con él. En los niños, la tendencia es a la identificación dispersa, de modo que en ellos la agresividad y la erotización promueve la identidad confusa y no se logra focalizar el objeto al que tiende.

\section{DE LA ESCUELA SUBSIDIARIA A LA SUSTITUTA}

La escuela subsidiaria estaba claramente tipificada en la antigüedad, abría sus puertas a niñas o niños que provenían de familias numerosas, donde la experiencia de tratar con hermanos de distinto sexo enseñaba de manera natural las diferencias entre ellos. A la escuela iban a adquirir conocimientos y a aprender a ser amigos. El modelo de familia de los alumnos era semejante, la madre permanecía en la casa y se encargaba de hacer rendir los recursos que el padre aportaba.

El divorcio era un recurso extremo, por lo tanto, se luchaba por llevar la vida en paz y se evitaban aquellas situaciones que deterioraran una relación para toda la vida. Entonces se contaba con la figura materna y paterna estables, con virtudes que hacían grato el ambiente.

En la actualidad se espera mucho más de la escuela. Los educandos acarrean un lastre de inseguridad, muchas veces por falta del afecto que no encuentran en sus padres, porque ambos dedican buena parte de su tiempo al trabajo extradoméstico o por la ausencia de uno de los progenitores debido al divorcio, al abandono o a la suplantación de alguno de ellos. De los maestros se espera comprensión, paciencia y buen ejemplo para rehacer la figura femenina o masculina que no observan en sus progenitores.

La propaganda de la cultura de género encuentra eco cuando el modelo de alguno de los progenitores es negativo, en la intimidad aparece el rechazo a ese sexo y se busca el 
otro. Entonces se dan actitudes aberrantes que la sociedad admite. De allí que los educadores ahora tengan la responsabilidad, antes no imaginada, de rehacer en los educandos la figura del hombre o de la mujer, según el caso.

De este modo el papel de subsidiariedad, que inicialmente tuvo la escuela, se ha vuelto complejo. Por las abundantes carencias familiares aparece la necesidad de suplir para rehacer las personalidades de los educandos. Un aspecto especialmente relevante es el de la educación de la afectividad y del pudor, que salvaguardan el verdadero cariño.

\section{EL DESARROLLO DE LA SEXUALIDAD EN LAS DOS PRIMERAS DÉCADAS DE VIDA}

Para normar el criterio sobre la oportunidad de una escuela diferenciada según el sexo, hace falta explorar los datos que se tienen del desarrollo y descubrimiento del sexo desde la infancia hasta la adolescencia.

En la fecundación, cuando los 23 pares de cromosomas son X, sabemos que se trata de una niña. Si el padre aporta 22 cromosomas X y uno Y se engendra un niño. Por eso, desde el primer momento se da la diferenciación sexual. Un poco más adelante sucede lo que nos explica el doctor Ernesto Bolio y Arciniega:

(...) la séptima semana de gestación, el feto debe recibir un "baño hormonal", y en ese momento el cerebro, la estructura cerebral, se ve modificada por ese "baño" (...). Este "baño hormonal" —que produce la madre- define en las células una orientación sexual de la que carecerían sin el "baño", de hecho, algunas veces, por razones desconocidas aún, se producen más o menos hormonas y eso también influye en el feto ${ }^{3}$.

A los dos años, los pequeños descubren su cuerpo y las diferencias entre niñas y niños y asumen su propio aspecto ${ }^{4}$,

${ }_{3}^{3}$ Vicisitudes de la identidad sexual, en Revista ISTMO, No. 255, p.43.
${ }^{4}$ Cfr. ERIKSON, Eric H. Infancia y sociedad, pp.61 y 75. 
desde ese momento se identifican con su ser varonil o femenino de manera espontánea. En la pubertad ${ }^{5}$, los cambios hormonales y sus efectos en el cuerpo, llevan a afrontar de un modo radical el sentimiento de la identidad sexual. Entonces los intereses y las conductas de los adolescentes están fuertemente relacionados con la sexualidad.

El trato del niño con el padre o la madre es muy importante para empezar a construir la relación con personas del mismo o del otro sexo, esto ayudará a que en la adolescencia la identificación con el propio sexo se asuma sin ningún tipo de dificultad.

Los problemas ${ }^{6}$ que actualmente pueden presentarse en los adultos, y que afectan la imagen que de ellos tengan sus hijos repercutiendo en la aceptación de la identidad, pueden deberse al divorcio de los padres y a la sustitución de los progenitores; a la resistencia del hombre al trabajo de la mujer fuera del hogar y al éxito que de ello se derive, que en ocasiones es superior al de él; a la reducción del tiempo que la madre dedica a la familia, por variar sus roles en la vida cotidiana, y a que muchas veces el padre tiene más presencia que ella en el hogar, etcétera.

El efecto más frecuente de todo lo anterior es la ruptura de la armonía entre los tres aspectos del sexo’: biológico, psicológico y social, y las repercusiones en la conducta sexual. El primero es el cromosómico; el segundo se relaciona con las vivencias psíquicas y se manifiesta en la toma de conciencia de pertenecer a un determinado sexo; el último es el asignado a una persona según la perciban los demás. Cuando estos aspectos se desarticulan, los adolescentes adoptan conductas inadecuadas porque se resisten a identificarse con su sexo biológico y enfocan la sexualidad de manera antinatural. Esto puede agravarse ya que en estas circunstancias:

${ }^{5}$ Cfr. MUSSEN, Paul H., CONGER, John J. y KAGAN, Jerome. Desarrollo de la personalidad en el niño, pp.436 y ss.

${ }^{6}$ Cfr. GONZÁLEZ NÚÑEZ, José de Jesús (Compilador). Los cambios del hombre frente a la metamorfosis de la mujer.

${ }^{7}$ Cfr. BURGGRAF, J. ¿Qué quiere decir género?, pp.16-19. 
(...) el desarrollo sexual no es completo y no ha madurado hasta el pleno descubrimiento de su meta: el sexo opuesto. En este momento del crecimiento, objetivos y situaciones variadas, humanos o no humanos, pueden asociarse en la imaginación con el despertar de sentimientos eróticos todavía indefinidos: niños y adultos, pero también situaciones inanimadas o emocionalmente excitantes. La sexualidad de un adolescente en esta etapa podría denominarse bisexual, aunque no sería desacertado llamarla "multisexual" ${ }^{8}$.

\section{LA ESCUELA MIXTA}

Aunque en casi todas las culturas de la antigüedad se educó a las mujeres, la escolaridad siempre se polarizó hacia los niños debido a los esquemas sociales. Obviamente, cuando las oportunidades educativas son las mismas para el varón y la mujer, hace falta precisar la terminología y todo ello propicia un desconcierto que poco a poco se ha ido superando.

El planteamiento de fondo ante la propuesta de escuelas diferenciadas o mixtas responde a que, en algunos casos, se da mayor importancia a la personalidad humana y en otros se defiende la necesidad de dar al hombre y a la mujer las mismas oportunidades educativas?.

Antonio Ardillas y Jesús Puente al estudiar la coeducación ${ }^{10}$ señalan aspectos negativos y positivos. Entre los primeros, admiten la dificultad de establecer el justo equilibrio para desarrollar las cualidades femeninas y masculinas sin menoscabo de algunas, los educadores generalmente no tienen la suficiente preparación para enfrentar la coeducación, se dificulta la educación sexual y se fomentan las relaciones sexuales prematuras. Respecto a lo positivo señalan que se ofrece una formación humana integral pues lo normal es convivir con

\footnotetext{
${ }^{8}$ VAN DEN AARDWEG, Gerard J. M. Homosexualidad y esperanza, pp.57-58.

${ }^{9}$ Cfr. GARCÍA HOZ, Víctor. Principios de Pedagogía sistemática, pp.275-283.

${ }^{10}$ Cfr. Coeducación, STUDIUM, Madrid, 1970.
} 
mujeres y varones, se enriquecen con más variados enfoques, practican la caballerosidad y el pudor, destierran la timidez, se fomenta la igualdad de oportunidades.

Este estudio realizado hace poco más de treinta años no contaba con el actual deterioro del respeto entre las personas de diferente sexo ni con la permisividad de las manifestaciones de afecto que exacerban la sensibilidad. Todo ello con el apoyo de los medios de difusión que presentan, como modelo de vida, el de personas públicas que desconocen el significado de la fidelidad y del compromiso.

En Estados Unidos, país caracterizado por la defensa de la igualdad a ultranza, se ha iniciado un planteamiento que tal vez responda a la necesidad de evitar la promiscuidad y la procreación a edades tempranas y sin la garantía de formar una familia:

El respaldo a las escuelas de un único sexo supera las posiciones de los partidos políticos. En el senado norteamericano, los defensores van desde el republicano de Texas, Kay Bailey Hutchinson, a demócratas como Hillary Clinton de Nueva York y Edward Kennedy de Massachussets.

Associated Press, citando a Leonard Sax, responsable de la Asociación Nacional para la Implantación de Escuelas de un Único Sexo en la Educación Pública, informaba el 8 de mayo que ya existen 10 escuelas públicas de un único sexo, y dos más se espera que se abran en breve ${ }^{11}$.

\section{PROPUESTAS}

El panorama ofrece más sombras que luces, sin embargo, la postura que se plantea se apoya en la realidad de la naturaleza humana, en lo que es, imagen y semejanza de El que Es.

${ }^{11}$ BOLETÍN ELECTRÓNICO DEDICADO A LA DIFUSIÓN DE LA EDUCACIÓN FAMILIAR, LOS VALORES Y LA DEFENSA DE LA VIDA HUMANA DESDE LA CONCEPCIÓN HASTA LA MUERTE NATURAL, ¿Ha llegado la hora de las escuelas separadas por sexos?, No. 302, p.1. 
Por tanto, aunque es arduo el trabajo, la verdad está de nuestro lado.

La familia ha de recuperar su papel de primera educadora y la escuela tomar su postura de subsidiariedad. Para ello hace falta una mutua colaboración: ofrecer a los padres acertados criterios mediante "Escuelas para padres" donde se les ayude a resolver los problemas de los hijos y aconsejarles para que muestren una vigorosa y atractiva personalidad materna y paterna. De este modo ayudan a los hijos a adoptar sin problemas su propia identidad y el aprecio por el otro sexo.

...no se trata de que los varones sean más "masculinos" y las mujeres más "femeninas" (pero tampoco lo contrario), sino de que vivan más como "personas", lo cual significa con más originalidad, individualidad, autonomía, refiriéndose menos "a lo que se suele hacer" y a "lo que todos piensan", con creciente disposición de aceptar en libertad la responsabilidad de los propios pensamientos y sentimientos, juicios y actos ${ }^{12}$.

La preparación de los maestros ha de responder a las demandas de los hijos que no cuentan con el apoyo de un buen ambiente familiar; hay que fortalecerlos para evitar soluciones fáciles que a la larga provocarán problemas similares a los de los progenitores. Además, la personalidad de cada maestro ha de ser vigorosa para ejemplificar su respectivo sexo. De los docentes se espera fortaleza para ir en contra del ambiente permisivo y erotizado.

Ofrecer diversos tipos de escuelas: femeninas, masculinas y mixtas, para poder elegir la que más convenga a los educandos, previo estudio minucioso de la personalidad y el ambiente en donde se desenvuelven. Por ejemplo, si lo que un niño necesita es relacionarse con otros niños porque en su familia está rodeado de mujeres, la opción es un colegio

${ }^{12}$ BURGGRAF, Jutta. Hacia un nuevo feminismo para el siglo XXI, pp.49-50. 
masculino. Cada institución ha de tener muy claro el objetivo de la formación diferencial y conocer muy bien los momentos críticos donde se configura la feminidad y la masculinidad. Un medio para lograrlo es el de ofrecer una adecuada educación sexual en donde se responda a las siguientes preguntas: ¿Cómo hablar a los hijos de este tema? ¿Cuándo y cómo decirlo? ¿Quién debe decirlo? ¿Cuáles son los conceptos que ameritan aclaración? El doctor Álvaro Sierra Londoño afirma:

Mi condición de médico de niños, con alguna experiencia en educación y asesoría familiar, al lado de mi condición de padre de familia, me habilitan para afirmar sin temor a equívocos a todos los padres de familia que están seriamente preocupados por la educación sexual de sus hijos, que nadie puede ni debe hacerlo mejor que ustedes. Y en cuanto al medio educativo escolar, estén vigilantes y atentos, porque su función al respecto es de complementación frente a lo realizado por los padres. Sólo los padres han de encuadrar la sexualidad humana [en su auténtico eje]: el amor. Porque sólo ellos pueden avalarlo con la vida misma, su compromiso, su incondicionalidad y su capacidad para entregarse totalmente ${ }^{13}$.

Se trata de recuperar los valores sociales de la fidelidad y del respeto, como un reto que al unísono ha de establecerse en la familia y en la escuela, con la firme determinación de no perderlos nunca más.

${ }^{13}$ Apud TAMÉS, María Adela. La mujer y la crisis de la familia, p.91. 


\section{BIBLIOGRAFIA}

ARDILlAS, Antonio y PUENTE, Jesús. Coeducación, STUDIUM, Madrid, 1970.

BURGGRAF, Jutta:

- ¿Qué quiere decir género?, Ediciones Promesa, Costa Rica, 2001.

- Hacia un nuevo feminismo para el siglo XXI, Ediciones Promesa, Costa Rica, 2001.

ERICKSON, Eric H. Infancia y sociedad, 8ª edición, Ediciones Hormé, Buenos Aires, 1980.

GARCÍA HOZ, Víctor. Principios de Pedagogía sistemática, $3^{a}$. Edición, Ediciones RIALP, S. A., Madrid, 1966.

GONZÁLEZ NÚÑEZ, José de Jesús (Compilador). Los cambios del hombre frente a la metamorfosis de la mujer, Instituto de Investigaciones en Psicología Clínica y Social, A.C., México, 2000.

MUNSSEN, Paul H., CONGER, John J. y KAGAN, Jerome. Desarrollo de la personalidad en el niño, $4^{\underline{a}}$ reimpresión, Editorial Trillas, México, 1998.

TAMÉS, María Adela. La mujer y la crisis de la familia, Ediciones Promesa, Costa Rica, 1999.

BOLETÍN ELECTRÓNICO DEDICADO A LA DIFUSIÓN DE LA EDUCACIÓN FAMILIAR, LOS VALORES Y LA DEFENSA DE LA VIDA HUMANA DESDE LA CONCEPCIÓN HASTA LA MUERTE NATURAL, No. 302, Julio 2002.

REVISTA ALETHEIA. No. 20, México, 2001.

REVISTA ISTMO. Año 43, No. 255, Julio-agosto 2001.

VAN DEN AARDWEG, Gerard J. M. Homosexualidad $y$ esperanza, Ediciones Universidad de Navarra, S. A., España, 1997. 